\title{
The Effect of a Psychoeducational Course on Hypochondriacal Metacognition
}

\author{
Femke Marrit Buwalda - Theo K. Bouman • \\ Marijtje A. J. Van Duijn
}

Published online: 2 February 2008

(C) The Author(s) 2008

\begin{abstract}
Recently, it has become apparent that a metacognitive perspective may contribute to the understanding and treatment of psychopathology. In this study, the effect of a cognitive-behavioural psychoeducational group treatment for hypochondriasis on metacognitive aspects was examined. Furthermore, it was studied whether earlier found beneficial effects of the course on hypochondriacal complaints, depressive complaints and trait anxiety could be replicated. A total of 35 participants were randomized into either an immediate treatment group ( $n=20)$, or a waiting list control group $(n=15)$. The participants in the waiting list control group were enrolled in the treatment after a period of 6 weeks. Results showed that the course had an effect on all subscales of the Metacognition-Cognitions about Health Anxiety questionnaire. Furthermore, in line with previous findings, the course proved to be
\end{abstract}

F. M. Buwalda $(\bowtie) \cdot$ T. K. Bouman

Department of Clinical and Developmental Psychology, University of Groningen, Grote Kruisstraat 2/1,

9712 TS Groningen, The Netherlands

e-mail: F.M.Buwalda@rug.nl

T. K. Bouman

e-mail: T.K.Bouman@rug.nl

M. A. J. Van Duijn

Department of Sociology/Statistics and Measurement

Theory, University of Groningen, Grote Rozenstraat 31,

9712 TG Groningen, The Netherlands

e-mail: M.A.J.van.Duijn@rug.nl effective in decreasing hypochondriacal complaints, depressive complaints and trait anxiety. It may be concluded that cognitive-behavioural psychoeducational treatment, in which a metacognitive level is implicitly addressed, shows beneficial effect on metacognitive aspects and hypochondriacal complaints.

Keywords Hypochondriasis - Metacognition · Psychoeducation $\cdot$ Cognitive-behavioural intervention

\section{Introduction}

The central mechanism in people suffering from hypochondriasis is a relatively enduring tendency to misinterpret bodily symptoms, bodily variations, and other health-related information, as evidence of serious physical illness (Asmundson et al. 2001). According to the cognitive-behavioural hypothesis of health anxiety and hypochondriasis (Warwick and Salkovskis 1990), bodily signs and symptoms are perceived as more dangerous than they really are, and the chance of contracting a particular disease is believed to be more probable than it really is (Salkovskis 1989; Salkovskis and Warwick 1986; Warwick and Salkovskis 1989). This cognitive hypothesis accounts for the development of hypochondriasis, in that knowledge and past experiences of disease lead to the formation of specific 
assumptions about symptoms, disease and health behaviours. These assumptions will often lead to a confirmatory bias in the patient's thinking once a critical incident has resulted in the misinterpretation of bodily symptoms and signs as being indications of a serious disease. Mechanisms that are subsequently involved in the maintenance of hypochondriacal complaints are anxiety -resulting in physical arousal-, selective attention -such as the perception of normal bodily changes, and previously unnoticed bodily features-, and behaviour, designed to avoid, check for or exclude physical disease.

Metacognitive processes might explain how misinterpretation can play a continuing role in the maintenance of hypochondriasis. Metacognition is any knowledge or cognitive process that is involved in the appraisal, monitoring, or control of cognition (Flavell 1979). A distinction has been made between three components of metacognition: metacognitive knowledge, metacognitive experiences, and metacognitive control strategies (Wells 2000). These three components can help explain why metacognition has been hypothesized to play a part in emotional disorders. Metacognitive knowledge refers to the information that people have about their own cognitions, such as beliefs about the meaning of particular types of thoughts (such as worrying), and beliefs concerning the efficiency of memory and cognitive control (Wells 2000). Metacognitive knowledge can be explicit and accessible to patients. Examples of explicit metacognitive knowledge are: 'I cannot control my worrying', or 'If I think about cancer so much, I will get it'. However, metacognitive knowledge can also operate in an implicit way, and forms the rules or plans that guide processing, such as attention allocation (Wells 2000).

Secondly, metacognitive experiences include appraisals of the meaning of specific mental events, metacognitive feelings and judgements of the status of cognition (Wells 2000). Thirdly, metacognitive control strategies are the responses individuals make in controlling the activities of their cognitive system (Wells 2000). In clinical disorders, control strategies often consist of attempts to control the stream of consciousness.

It was hypothesized that metacognitive processes operate on two different levels, which are interrelated: the meta-level and the object-level (Nelson and Narens 1990). Information flows to and from both levels, and is called monitoring when the objectlevel informs the meta-level of its state, and control when the meta-level informs the object-level what to do next. It is thought that the meta-level is controlled and modified by feedback about the effectiveness of particular cognitive and behavioural strategies in relation to activated goals (Wells and Matthews 1994), thereby providing information about future preferred actions and cognitions.

The Metacognition Questionnaire (MCQ; Cartwright-Hatton and Wells 1997) was devised to assess individual differences in the aforementioned metacognitive aspects. The MCQ consists of several subscales: (a) positive beliefs about worry, referring to the extent to which a person feels that worrying is helpful, (b) negative beliefs about worry, concerning uncontrollability and danger, (c) cognitive confidence, such as confidence in attention and memory, (d) negative beliefs concerning the consequences of not controlling one's own thought, and (e) cognitive selfconsciousness, referring to the tendency to monitor one's own thoughts and focus attention inwards.

In recent studies investigating metacognitive aspects, several disorders were found to be influenced by metacognition. A study of depression (Papageorgiou and Wells 2003) showed a relationship between metacognition, rumination and depression. Furthermore, the study showed that positive beliefs about rumination could be associated with the activation of rumination. Furthermore, rumination appeared to lead to symptoms of depression, directly or indirectly, via negative metacognitive beliefs about the interpersonal and social consequences of ruminating.

Metacognition was also found to play a role in anxiety disorders. Obsessive compulsive disorder (Wells and Papageorgiou 1998) was found to be influenced by metacognition: both positive beliefs and negative beliefs about worry concerning themes of uncontrollability and danger were positively associated with proneness to pathological worry in obsessive compulsive disorder. All MCQ subscales were significantly and positively correlated with obsessional checking and obsessional thoughts. A relationship between generalised anxiety disorder and metacognitive aspects has also been shown (Wells and Carter 2001). Generalized anxiety disordered patients had higher meta-worry and negative metacognitive belief scores than social phobic patients, panic disordered patients, depressed patients and 
nonpatients. A most recent study showed a relationship between post-traumatic stress disorder and metacognitive aspects (Roussis and Wells 2006). In this study it was hypothesized that worry and specific positive and negative metacognitive beliefs would be positively associated with stress symptoms. Indeed, results showed that thought control strategies of worry, and positive and negative metacognitions were positively associated with stress symptoms.

As yet, it has only been studied once whether metacognitive aspects played a role in, or were related to, hypochondriacal complaints (Bouman and Meijer 1999). The researchers adapted the MCQ for use with hypochondriacal patients, resulting in the Metacognition-Cognitions about Health Anxiety (MCHA). This scale measures beliefs and attitudes in connection with anxious thoughts about health, illness, and disease. Specific metacognitive areas, captured in the instrument's subscales, are uncontrollability and interference, self-consciousness, responsibility, and positive and negative consequences of thinking about illness. Results of the study showed that metacognition played a part in hypochondriasis: hypochondriacal patients specifically had many worrisome thoughts about their health. On a metacognitive level they were concerned about the lack of control, and the excess of interference they experienced in relation to their illness-worries. They proved highly aware of their own thoughts and worries, and content-specific meta-worry appeared to be the best predictor for hypochondriasis. Hypochondriacal patients did not score higher on positive beliefs and responsibility than healthy controls and psychology students. However, because only 14 patients suffering from hypochondriasis participated, and this study was the first to examine the relationship between metacognition and hypochondriasis, it is too early to conclude as yet that these two metacognitive aspects do not play a role in hypochondriasis. Furthermore, clinical experience suggests that the metacognitive aspects that are measured using the MCHA may well play a role in the maintenance of hypochondriasis.

For one, uncontrollability is manifest in that hypochondriacal patients tend to regard their cognitions and worries about illness as almost impossible to control. Furthermore, they seem to believe they have the responsibility not to get a serious disease, or to prevent an already contracted disease from getting worse, for example by visiting physicians and seeking reassurance from their partners or others. Also, hypochondriacal patients are known to display selfconsciousness: when worrying about a disease they report to find it very difficult to focus their attention on anything else than their bodily sensations.

Hypochondriacal patients state that they often try to stop thinking catastrophically, but that they are somehow not able to do so, perhaps because the ways in which they attempt to control their cognitions are unhelpful. Firstly, as a result of the negative consequences of cognition perhaps, they try to force themselves to stop thinking about illness, and not be confronted with illness. Therefore, people suffering from hypochondriasis tend to avoid disease related situations and information. Secondly, hypochondriacal patients allow themselves to ruminate about their health, possibly as a result from the positive consequences they believe thinking about disease has.

An important question is how those metacognitive aspects that maintain emotional disorders can be addressed in clinical practice. One way to do so is by developing metacognitive treatments, which explicitly focus on metacognition. This has been done by Wells (1999, 2000), at first for GAD, and later for post-traumatic stress disorder (Wells and Sembi 2004). In metacognitive treatment, the emphasis lies on eliciting and modifying negative and positive beliefs about worrying. Furthermore, alternative nonworry-based strategies for appraising and dealing with threat are developed as part of the treatment. Participants are made specifically aware of their metacognitions and how to change them.

However, specific metacognitive interventions might not be necessary for every disorder in which metacognitive aspects play a role, because metacognitions may also implicitly be influenced by certain treatments. Cognitive-behavioural psychoeducational treatment is one of the treatment forms in which this may happen, because participants are taught that their cognitions and behaviours are not as uncontrollable and dangerous as they seem. These new insights might lead to a change in metacognition, in terms of participants gaining control over their thoughts and actions without having discussed metacognitive aspects. Furthermore, during cognitive-behavioural psychoeducational treatment participants are taught how to monitor, appraise and control their thoughts and behaviour in a different and more adaptive way, possibly leading to different patterns of behaviour and a different perception on worrying about illness as well. 
In recent years, short-term psychoeducational courses based on the cognitive-behavioural approach have shown to be effective in reducing hypochondriacal complaints (Avia et al. 1996; Barsky et al. 1988; Bouman 2002; Buwalda et al. 2007; Stern and Fernandez 1991). In the present study, we investigate whether the Dutch course, called 'Coping with health anxiety' is effective in changing metacognitions.

The research has two main aims: the first is to examine whether the 'Coping with health anxiety' course can produce a change in several aspects of metacognition, operationalized as the MCHA subscales, and hypochondriacal complaints. It is hypothesized that all measured aspects of metacognition (uncontrollability and interference, self-consciousness, responsibility, and positive and negative consequences of thinking about illness) decrease after the course, having been implicitly addressed through the psychoeducational treatment. The second goal of the study is to replicate earlier found beneficial effects of the course on hypochondriacal complaints, depressive complaints, which hypochondriacal patients often also suffer from, and trait anxiety. The course is expected to positively affect hypochondriacal complaints, depressive symptomatology and trait anxiety.

\section{Method}

Recruitment, Screening and Randomization

Participants were recruited by notifying the local press, local radio networks, General Practitioners (GP's), and low threshold general health care facilities a few times over a period of 4 years (2002-2005). The course was open to self-referral, and was introduced as a way of learning how to handle health anxiety, and of gaining insight into hypochondriacal complaints.

Potential participants were screened for psychopathology using a structured $30 \mathrm{~min}$ telephone interview. This interview is a condensed version of the Anxiety Disorders Interview Schedule (Bouman et al. 1997; DiNardo et al. 1994) in which only the main criteria for DSM-IV (APA 1994) somatoform, anxiety and mood disorders were screened, with a specific emphasis on hypochondriacal complaints. An example of how only the main criteria of anxiety disorders were screened is: when inquiring into generalized anxiety disorder, the interviewer merely asked whether or not there were multiple things the participant worried about, whether this worrying had increased over the last 6 months, and whether it was difficult to stop worrying about these things. Participants were also asked about previous psychological treatment. The interview led to an evaluation of the presence or absence of symptoms of any of the disorders mentioned above.

Inclusion criteria were: (1) the presence of a DSMIV diagnosis of hypochondriasis, (2) being over 18 years old, (3) having active command of the Dutch language, and (4) being willing to participate actively in the course. Exclusion criteria were: (1) the presence of other DSM-IV Axis I disorders more prominent than hypochondriasis, (2) the presence of a serious somatic disease as the focus of the health anxiety, and (3) a previous or concurrent cognitivebehavioural treatment for hypochondriasis. The participants using psychotropic medication when entering the study $(n=13)$, were asked to keep their dosage constant for the sake of the study.

Informed consent was obtained at the end of the telephone interview, by first giving potential participants information about the nature of the study, and then informing them they were free to stop their participation in the research at any given time, without this interfering with their participation in the course. They then were asked if they agreed to these terms. None of the candidate participants refused to participate in the study.

Participants were randomly assigned to either the immediate treatment condition, or the waiting list condition, by order of application: once 6-8 participants had applied, and had been included, either an immediate treatment- or a waiting list group started. The waiting list period lasted 6 weeks, after which period participants were enrolled in treatment. The randomisation was undertaken irrespective of patient characteristics, and was performed by the first author. The courses were taught at a Home Care organization, as well as at the Department of Clinical Psychology of the University of Groningen, where the research was conducted.

\section{Participants}

Of the 57 people interested in the course, 35 participants were randomized to the course, implying 
that 22 aspiring participants declined to participate. Two of them preferred individual treatment, 4 of them were already treated elsewhere, one was unable to attend the course, three of them decided that their complaints were not severe enough to require treatment, three of them were suffering primarily from other complaints, another three could not be reached after the initial contact, five candidates had lost their interest after the telephone interview, and one candidate's proficiency in Dutch was insufficient.

The 35 participants were randomised into six groups: three immediate treatment groups (total $n=$ 20 ), and three waiting list groups (total $n=15$ ), of 5-8 participants. Four (11.4\%) participants dropped out of the course, after the first or following sessions (two in the immediate treatment group, and two in the waiting list group), of whom one participant stopped coming after the second session and was impossible to contact thereafter, one could not attend because of obligations at work, one stopped because a benign cause of her bodily symptoms was found and she decided she did not need the course anymore, and one felt the course did not suit her problems. In the immediate treatment condition, 18 participants completed the course, and of the waitlist condition, 13 participants did so. Of the 31 course completers, 26 participants returned the post assessment (14 of the immediate treatment condition, and 12 of the waiting list condition). A total of 26 completers returned the 1 month follow-up (14 participants of the immediate treatment condition, and 12 of the waiting list condition), and 22 completers returned the 6 month follow-up (11 participants of the immediate treatment condition, and 12 of the waiting list condition).

Of the participants, $21(60 \%)$ were female, and the mean age was 38.2 years $(S D=10.6)$. A total of 23 participants $(65.7 \%)$ were cohabitating or married. Eleven $(31.4 \%)$ of the participants had a high (academic or professional) educational level, 15 (42.9\%) of the participants had a medium (higher secondary) level of education, and $8(22.9 \%)$ had a low (lower secondary) educational level. Mean duration of hypochondriacal complaints is 12.3 years ( $S D=10.3$, range 6 months -41 years). Chi-square testing and $t$-tests showed no significant differences between the conditions regarding either of these demographic variables.

Only participants who, during the telephone diagnostic interview, stated that their primary complaint was hypochondriasis and that they specifically required help with regard to these complaints, were included in this study, and were informed that hypochondriacal complaints would be the sole focus of the course. However, comorbid complaints were also evaluated shortly during the diagnostic telephone interview. A total of 17 participants suffered to some extent from panic attacks, and 12 from general anxiety complaints. Six participants reported agoraphobic complaints. Furthermore, 13 participants reported some form of specific phobic complaint, whereas 8 suffered from social phobic complaints. Three participants suffered from a moderate form of obsessive compulsive complaints. Regarding depressive episodes, a total of 27 participants had suffered from these, either at time of intake or in the past. Most participants stated that these depressive complaints were related to their hypochondriacal complaints. We have no information regarding comorbidity of 3 participants. A substantial number of participants suffered from symptoms of more than one anxiety- or depressive complaint. Between the participants of the immediate treatment- and the waiting list condition there were no significant differences with regard to the occurrence of comorbid complaints, according to chi-squared test.

\section{Procedure}

The course 'Coping with health anxiety' was implemented as a six 2-hour session format, each of those consisting of a mixture of mini-lectures, demonstrations, video illustrations, focused group discussions and brief exercises. The course's theory is based on the cognitive-behavioural model by Warwick and Salkovskis (1990), which describes several mechanisms that are subsequently involved in the maintenance of hypochondriacal complaints. These mechanisms are bodily symptoms, cognitions (about these symptoms), anxiety (resulting in physical arousal), selective attention (such as the perception of normal bodily changes, and previously unnoticed bodily features), and behaviour, designed to avoid, check for, or exclude, physical disease. Every session of the course is based on one such mechanism, and consists of detailed explanation and practical exercises. In order to increase personal relevance and active mastery of the information provided, the 
facilitators try to elicit as many examples and responses as possible from the participants themselves. Sessions one to five are followed by brief, optional, homework assignments. A booster session is held 4 weeks after session six. The course is described in more detail elsewhere (Bouman 2002; Bouman and Buwalda in press).

Each group was coached by two facilitators. The group of facilitators consisted of one PhD-student, with 3 years of experience with both individual CBT for hypochondriasis, and the course 'Coping with health anxiety' (first author), and several graduate students of clinical psychology (all females, in their early twenties). All facilitators had some experience with individual cognitive-behavioural treatment for hypochondriasis; some had previous experience with coaching courses. A detailed session-by-session manual was provided and used by the facilitators, and they received a 3 -h training in teaching the course, provided by the first author. During the training, the manual was discussed and facilitators were taught how to handle certain situations that may arise during the course, such as the participants not paying attention, or one participant seeking more attention and guidance by the facilitators than the others. The facilitators were supervised weekly by the first author, to discuss progress, specific content of the sessions, and to detect and solve possible problems. These supervision sessions also served as a way to qualitatively assess adherence to the manual. Having two facilitators teaching the course served as a safeguard for treatment fidelity, as did having them write down detailed session reports.

\section{Measurements}

Repeated measures were taken pre-treatment, posttreatment, at 1 month after the course had ended, and at 6 months after the ending of treatment.

\section{Primary Outcome Measures}

\section{Hypochondriacal Complaints}

The Groningen Illness Attitude Scale (GIAS; Bouman 2002; Visser 2000) is a 42-item self-report questionnaire that measures four aspects of hypochondriasis: 'disease conviction' (15 items; $\alpha=0.92$ ), 'bodily symptoms and complaining' (12 items; $\alpha=0.88$ ), 'health anxiety and thanatophobia' (8 items; $\alpha=0.85$ ), and 'checking and avoidance behaviour' (7 items; $\alpha=0.71$ ) (Bouman 2002). The GIAS is based on the Illness Attitude Scales (Kellner 1986) and the Whitely Index (Pilowsky 1967). The applicability of each item during the seven days prior to assessment is scored on a 5-point scale (from $1=$ 'never', to $5=$ 'nearly always'). The questionnaire has satisfactory discriminative validity, and strong convergent validity (Visser 2000). In this study, the analyses will be done using the total scale of the GIAS.

\section{Metacognition}

The Metacognition-Cognitions about Health Anxiety (MCHA; Bouman and Meijer 1999) is a questionnaire containing 27 items measuring several components of metacognition. Items are scored on a scale from 1 ('do not agree') to 4 ('agree very much'). The 5 scales are: (a) Uncontrollability and interference of illness thoughts (12 items, e.g. 'I find it hard to ignore thoughts about serious diseases'; $\alpha=0.93$ ), (b) Cognitive self-consciousness (4 items, e.g. 'I am very aware of the way I think about illness'; $\alpha=0.74$ ), (c) Responsibility (3 items, e.g. 'I can protect myself from getting a serious illness by thinking about this a lot'; $\alpha=0.70$ ), (d) Negative consequences ( 5 items, e.g. 'I believe I can make myself sick by worrying about illness'; $\alpha=0.70$ ), and e) Positive beliefs ( 3 items, e.g. 'Worrying about diseases helps me to cope with my fear of them'; $\alpha=0.62$ ). The moderate internal consistencies of all but the first subscale are probably due to their moderate length.

\section{Secondary Outcome Measures}

\section{Depressive Complaints}

Beck's Depression Inventory (Beck et al. 1979; Dutch version: Bouman et al. 1985) measures the severity of depressive symptoms and consists of 21 groups of 4 statements describing depressive symptoms, from which the patient chooses the most applicable. Cronbach's $\alpha$ 's of this measure ranged 
from .73 to .92 within patient groups (Bouman et al. 1985).

\section{Trait Anxiety}

The trait scale of the Dutch authorized version of the State-Trait Anxiety Inventory (Dutch version: van der Ploeg et al. 1980) was used. This scale consists of 20 items and measures inter-individual differences in anxiety. The trait-scale has a Cronbach's $\alpha$ ranging between .91 (for college students) and .93 (for a patient normgroup) (Van der Ploeg et al. 1980).

\section{Results}

\section{Analytic Plan}

The effect of the course on metacognitive aspects over time was analysed using multilevel analysis. In contrast to standard methods for analysing repeated measurements, multilevel analysis does not require data on all measurements for each individual, but uses all available observations. In addition to some drop-out in the study described before, missing data occur due to the setup of the study: participants in the waiting list condition have one (earlier) extra measurement in comparison to participants in the immediate treatment condition. The structure of the data is depicted in Figure 1, where the measurements of participants in the waiting list and treatment condition are aligned at $\mathrm{t} 2$, the start of the course. Time $\mathrm{t} 1$ indicates the first measurement, only available for participants in the waiting list condition. In Fig. 1 also a few cases of dropout are visible, indicated with open triangles and circles for dropout before treatment. Dropout later in the study is apparent when individual lines are discontinued at for instance $\mathrm{t} 3$, indicating that no further measurements are available for follow-up after the course.

In multilevel analysis of repeated measurements, the observations (level 1) are nested in individuals (level 2). A first step in the analysis is to establish the covariance structure of the repeated measurements (comparable to the choice between the univariate or multivariate approach in repeated measures analysis of variance). Theoretically, a third level could have been included in the model, representing the variable
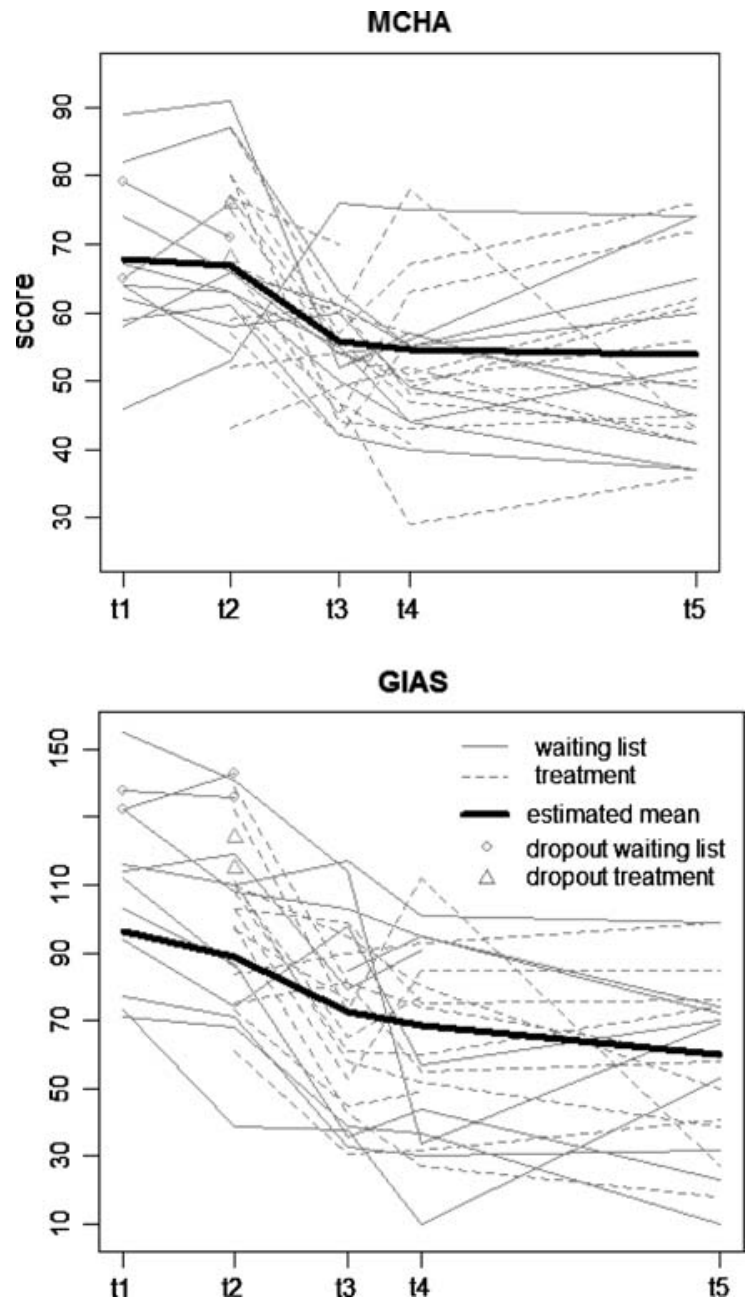

Fig. 1 The scores of all participants on metacognitive aspects and hypochondriacal complaints

'group'. However, due to the small amount of groups in this study, this was not a feasible option.

Multilevel models were estimated for the subscales of the MCHA and its total scale to investigate the effect of the course on metacognitive aspects over time, using dummy variables for the first measurement for the waiting list condition (t1) and for the measurement immediately after the course ( $\mathrm{t} 3)$ and the two followup measurements ( $t 4$ and $t 5)$, thus using the measurement at the start of the course as the reference time for both waiting list and immediate treatment conditions. Moreover, the difference between the immediate treatment condition and the waiting list condition was tested and left out of the model if not significant. 
To check whether participants with complete assessments differed from participants with missing data, the dummy variables for attrition based on the number of returned questionnaires was inserted in the multilevel models for every outcome measure. The dummy variable pertained to drop outs of the course, completers who returned one assessment, completers who returned three assessments, completers who returned a variable number of assessments in a random fashion (e.g. no pre-assessment, but followup one and two), and completers who returned all assessments. None of the completers returned two assessments, therefore this option is not included as a dummy variable. Only significant missing data dummy variables were retained in the model. The effect of biographical variables age, gender, and level of education was also investigated in the multilevel analysis. Non-significant variables were left out of the multilevel model.

The same model setup and selection was used for estimating the course effect on the GIAS measuring hypochondriacal complaints, the BDI measuring depressive complaints, and for the STAI measuring trait anxiety.

The statistical significance of single fixed effects is tested by approximate $t$-tests (Snijders and Bosker 2000), of which two-sided $p$-values are reported. In addition, the effect sizes of the post versus pre assessment difference, defined as the estimated difference between pre and post assessment, standardised using the estimated total standard deviation (Raudenbush 1997).

Multilevel Analysis Model Selection Results for All Dependent Variables

A multilevel model with just a level 1 (withinsubject) variance and a level 2 (between-subjects) variance was adequate for all dependent variables. This model corresponds to a compound symmetry model, assuming constant measurement variance and correlation between measurements over time. Both between- and within-subject variances for all variables are relatively large, which is also apparent from Fig. 1 with a large spread of lines (between subjects) and rather jagged individual lines (within-subjects). The between individual variance of all subscales was smaller than the measurement variance, meaning that scores of individuals differed less between subjects than within subjects. Only for subscales negative consequences and positive beliefs both types of variance did not differ substantially, indicating a resemblance in the pattern of fluctuation over time within individuals and between individuals.

When inserted into the models of all outcome measures (MCHA, GIAS, STAI, and BDI), only the drop out indicator showed an effect on the GIAS and was therefore included in the model of this measure. None of the biographical variables (age, gender, and level of education) had a significant effect and were therefore left out of the models.

The scores on the dependent variables at any time of assessment in the immediate treatment group did not differ from those in the waiting list control group, therefore condition as a variable was removed from the models.

\section{The Effect of the Course on Metacognition}

The estimates of the obtained multilevel models for metacognition and its subscales are given in Table 1 .

\section{Uncontrollability}

The waiting list period did not influence the uncontrollability subscale significantly $(t=-0.3, p>$ $0.25)$, but the course did: at post-assessment the mean score on uncontrollability decreased significantly $(t=-4.5, p<0.00)$. These scores decreased further at one month follow-up, and at six months follow-up. A large effect size between the second pre-assessment of the waiting list control group and the pre-assessment of the immediate treatment group, and post-assessment, was found.

\section{Cognitive Self-consciousness}

The mean score on this subscale did not decrease significantly during the waiting period. The first significant results were found at post-assessment $(t=-2.3, p<0.02)$. Scores increased slightly at the 1 month follow-up, but decreased further at the 6 months follow-up assessments. The effect size of the treatment was medium for this subscale. 


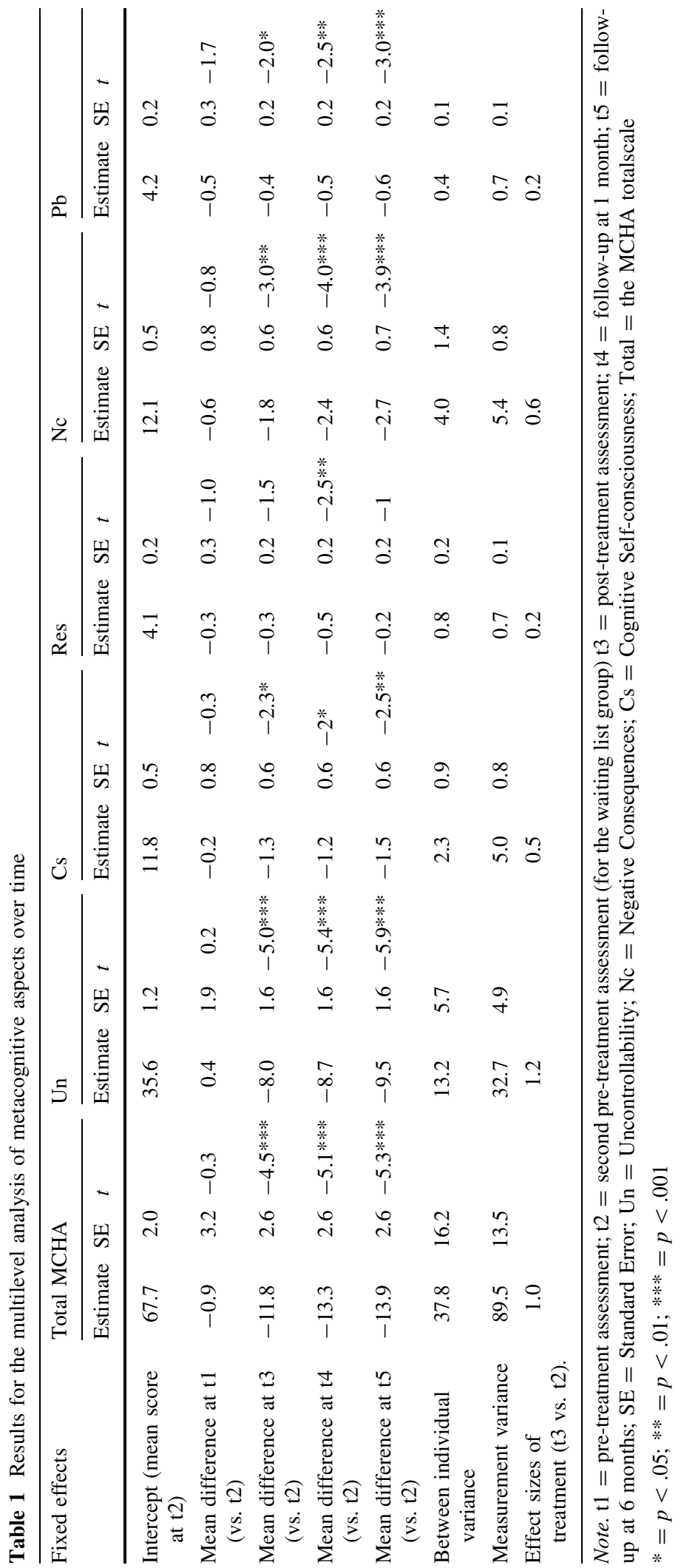


Responsibility

Scores on the responsibility subscales did not decrease significantly until the 6 months follow-up $(t=-2.5, p<0.01)$. The effect size of the treatment was small.

\section{Negative Consequences}

Table 1 shows that after the waiting list period, the mean score on this scale did not change. At postassessment, the mean score decreased significantly $(t=-3.0, p<0.00)$. At 1 month follow-up, the scores decreased further, to stabilise at 6 months followup. A medium effect size of the treatment was found.

\section{Positive Beliefs}

After the waiting list period scores did not change significantly, but they did at post-assessment $(t=$ $-2.0, p<0.05)$. Scores decreased further at the 1 month follow-up and the 6 months follow-up. Only a small effect size of the course was found (Table 2).

The change over time of total scale of the MCHA is in accordance with its subscales and is therefore not discussed in detail.
The Effect of the Course on Hypochondriacal Complaints, Depressive Complaints and Trait Anxiety

\section{Hypochondriacal Complaints}

During the waiting list period, no significant decrease in scores occurred $(t=1.3, p>0.15)$. At post-assessment, scores did decrease significantly ( $t=-4.7, p<0.00)$, and scores decreased further at 1 month and 6 months follow-up. Participants in the waiting list group did not differ from those in the immediate treatment group with regard to their scores on the GIAS. Being a drop out did have an effect on the results of the GIAS $(t=2.6, p<0.01)$ and was therefore included in the final model of this measure.

\section{Depressive Complaints}

BDI scores remained stable during the waiting list period ( $t=0.8, p>0.20$ ), but at post-assessment, scores decreased significantly $(t=-5.6, p<0.00)$. The scores decreased further at both follow-up assessments.

Table 2 Results for hypochondriacal complaints, depressive complaints and trait anxiety over time

\begin{tabular}{|c|c|c|c|c|c|c|c|c|c|}
\hline \multirow[t]{2}{*}{ Fixed effects } & \multicolumn{3}{|l|}{ GIAS } & \multicolumn{3}{|l|}{ BDI } & \multicolumn{3}{|l|}{ STAI } \\
\hline & Estimate & SE & $t$ & Estimate & SE & $t$ & Estimate & SE & $t$ \\
\hline Intercept (mean score at t2) & 96.6 & 4.8 & & 13.7 & 1.1 & & 52.9 & 1.5 & \\
\hline Mean difference at $\mathrm{t} 1$ (vs. $\mathrm{t} 2$ ) & 7.9 & 6.2 & 1.3 & 1.1 & 1.3 & 0.8 & 1.7 & 1.9 & 0.9 \\
\hline Mean difference at $\mathrm{t} 3$ (vs. $\mathrm{t} 2$ ) & -24.0 & 5.1 & $-4.7 * *$ & -5.6 & 1.0 & $-5.6 * *$ & -7.5 & 1.6 & $-4.7 * *$ \\
\hline Mean difference at $\mathrm{t} 4$ (vs. t2) & -28.1 & 5.0 & $-5.6 * *$ & -5.9 & 1.0 & $-5.9 * *$ & -7.7 & 1.5 & $-5.1 * *$ \\
\hline Mean difference at $\mathrm{t} 5$ (vs. t2) & -36.4 & 5.2 & $-7.0 * *$ & -8.1 & 1.1 & $7.4 * *$ & -10.8 & 1.6 & $-6.8 * *$ \\
\hline Drop out & 30.7 & 12.0 & $2.6^{*}$ & & & & & & \\
\hline Between individual variance & 355.5 & 113.4 & & 28.0 & 7.9 & & 46.6 & 13.9 & \\
\hline Measurement variance & 331.5 & 50.7 & & 13.6 & 2.1 & & 31.3 & 4.8 & \\
\hline Effect size pre-post & 0.9 & & & 0.9 & & & 0.8 & & \\
\hline
\end{tabular}

Note. $\mathrm{t} 1=$ pre assessment 1 of the waiting list group; $\mathrm{t} 2=$ pre assessment 2 of the waiting list group + the pre assessment of the immediate treatment group; $\mathrm{t} 3=$ post assessment; $\mathrm{t} 4=1$ month follow up assessment; $\mathrm{t} 5=6$ months follow up assessment. GIAS = Groningen Illness Attitude Scale; BDI = Beck's Depression Inventory; STAI = the trait scale of the Spielberger's State Trait Anxiety Inventory

$*=p<0.01 ; * *=p<0.00$ 


\section{Trait Anxiety}

The STAI did not show a significant decrease after the waiting period $(t=0.9, p>0.15)$. At postassessment, trait anxiety decreased significantly $(t=-4.7, p<0.00)$. The decrease continued at the one and 6 month follow-ups.

\section{Discussion}

The present study aimed to (a) examine whether the course 'Coping with health anxiety' could produce change in metacognitive aspects, and (b), whether the course's earlier found beneficial effects on hypochondriacal complaints, depressive symptomatology and trait anxiety would be replicated.

Results with regard to the first question showed that all metacognitive factors decreased during the course and persisted during the follow-up period, and that the course outperformed the waiting list period. This was the first time that a change in metacognitive aspects during a short-term, cognitive behavioral psychoeducational course was studied.

The large decrease (with an effect size of 1.2) in perceived uncontrollability indicates that participants found it much less difficult to stop worrying about illness after the course. They also changed their view on how abnormal it is to fear illness, for example by scoring lower on questions such as 'I often think there is something wrong with my way of thinking because I find it difficult to stop thinking about illness'.

The medium-sized decrease (effect size of 0.5) in cognitive self-consciousness indicates that people tended to focus less on their thoughts, and felt less need to critically examine their thoughts about illness after the course. This effect might be due to a change in selective attention, because participants were taught that they focus their attention on their bodies and illness-related cues, and how they can learn to control and change this. Furthermore, when health anxiety has decreased, examining thoughts about disease is no longer required.

A medium to large change (with an effect size equal 0.6) was found for negative consequences, which indicates that people were no longer as worried about the consequences of not being able to stop thinking about illness (e.g. going crazy), or actually contracting a serious illness by thinking about it constantly after the course. Because the participants were able to discuss their beliefs with other participants, and with the course's facilitators, they realized that having hypochondriacal metacognitions, and hypochondriacal cognitions, is not necessarily a sign that they are going crazy. Furthermore, the issue of whether it is possible to contract a serious disease just by thinking about the disease (which is an example of what we call 'magical thinking') was discussed specifically during the course.

Small effects were found on the subscales responsibility and positive beliefs (with effect sizes of 0.2 for both subscales), the first only at the 6 months follow-up. Similar to the findings by Bouman and Meijer (1999), who compared (a smaller number of) hypochondriacal subjects healthy controls and psychology students, the scores of our participants on these scales were not very high. Although we did find a significant change over time, the relationship between responsibility and positive belief and hypochondriasis is as yet unclear and should be studied further.

Through the course and its exercises, the participants learned how to cope actively with their health anxiety and to gain more control over their hypochondriacal complaints. Because the discussions during the course were specifically about cognitions, in terms of automatic thoughts and beliefs, but not about metacognitions, the alteration of metacognitive aspects as described above might be called implicit instead of explicit.

Overall, the findings regarding the decrease of metacognitive factors over time are in line with results found in earlier, smaller, studies in which metacognition changed during treatment. Wells and Sembi (2004) found that, with a treatment focussing on metacognition, PTSD symptoms decreased. However, their treatment specifically targets metacognitive factors, with an emphasis on eliciting and modifying negative and positive beliefs about worrying, and the development of alternative non-worry-based strategies for appraising and dealing with threat.

The treatment used in the present study did not explicitly focus on specific metacognitions participants might have. Instead, it has a format in which hypochondriacal complaints are normalized and presented as controllable, thereby probably changing implicit negative metacognitions about hypochondriacal complaints and cognitions. However, it is as yet 
unclear whether a treatment that specifically targets hypochondriacal metacognition could be even more beneficial. Therefore, it would be interesting to compare a metacognitive treatment for hypochondriasis to the short-term psychoeducational course 'Coping with health anxiety'.

With regard to the second research question, we found that the course also had a beneficial effect on hypochondriacal complaints. Furthermore, depressive complaints and state anxiety decreased over time. These findings were in line with earlier studies of the 'Coping with health anxiety' course (Bouman 2002; Bouman and Polman 2007; Buwalda et al. 2007).

This study has several limitations. One is the generalizability of the findings, with regard to both the change in metacognitive aspects and hypochondriacal complaints. The participants of this study were self-referred, and may differ from patients found in general mental health care in terms of severity of complaints and general functioning.

Another limitation is the occurrence of missing data in this study. It is impossible to make statements about the participants who did not return their questionnaires. Although we found that they did not differ from those who had returned all assessments with regard to their pre-assessment it still should be studied why participants decide not to return their measurements and how they could be motivated to return all assessments. We found that dropouts (participants who did not complete the course) had higher GIAS scores at the first measurement. They did not score higher on MCHA and its subscales. Because there were also successful completers with high GIAS scores on their pre-assessment, we cannot conclude that the course 'Coping with health anxiety' is not suitable for people with more severe hypochondrial complaints. However, they may have a lower probability to complete the course. Whether this is due to the hypochondrial complaints or to other related complaints is unclear as yet.

Additionally, there is is the lack of data on comorbidity of the participants. Due to the diagnostic instrument that was used in this study it was not possible to determine whether participants were eligible for concurrent diagnoses, as we only acquired information of depressive and anxiety symptomatology. Comorbidity information should be assessed more thoroughly in future studies, and at postassessment and follow-ups it should be measured whether participants still fulfill the diagnostic criteria of hypochondriasis.

Unfortunately, the design of the study does not allow a causal analysis of the data to determine whether metacognitive aspects or hypochondrial complaints were affected most by the course, and whether hypochondrial complaints were influenced by metacognition or vice versa. We can conclude however, that the higly correlated scale scores improved simultaneously during the course and that this improvement continued at least until 6 months after the course. It seems worthwhile to study the causal mechanism of the improvement further in a future study.

In conclusion, this study has shown that a cognitive-behavioural psychoeducational group course can produce change in both hypochondriacal metacognition and hypochondriacal complaints, and that the decrease in several aspects of metacognition concurs with a reduction of hypochondriacal complaints.

Acknowledgements The authors would like to thank Prof. Dr. P. Spinhoven for his comments on the limitations of causal analysis for this study.

Open Access This article is distributed under the terms of the Creative Commons Attribution Noncommercial License which permits any noncommercial use, distribution, and reproduction in any medium, provided the original author(s) and source are credited.

\section{References}

American Psychiatric Association (1994). Diagnostic and statistical manual of mental disorders (4th ed.) DSM-IV. Washington: APA.

Asmundson, G. J. G., Taylor, S., \& Cox, B. J. (2001). Health anxiety: Clinical and research perspectives on hypochondriasis and related conditions. Chichester: John Wiley and Sons Ltd.

Avia, M. D., Ruiz, M. A., Olivares, M. E., Crespo, M., Guisado, A. B., Sánchez, A., \& Varela, A. (1996). The meaning of psychological symptoms: Effectiveness of a group intervention with hypochondriacal patients. Behaviour Research and Therapy, 34(1), 23-31.

Barsky, A. J., Geringer, E., \& Wool, C. A. (1988). A cognitiveeducational approach for hypochondriasis. General Hospital Psychiatry, 10, 322-327.

Beck, A. T., Rush, A. J., Shaw, B. F., \& Emery, G. (1979). Cognitive therapy of depression. New York: Guilford.

Bouman, T. K. (2002). A community-based psychoeducational group approach to hypochondriasis. Psychotherapy and Psychosomatics, 71, 326-332.

Bouman, T. K., \& Buwalda, F. M. (in press). A psychoeducational approach to hypochondriasis: Background, 
content and practical guidelines. Cognitive and Behavioral Practice.

Bouman T. K., De Ruiter C., \& Hoogduin, C. A. L. (1997). The telephone interview: A condensed version of the anxiety disorders interview schedule. University of Groningen: Internal publication.

Bouman, T. K., Luteijn, F., Albersnagel, F. A., \& van der Ploeg F. A. E. (1985). Enige ervaringen met de Beck depression inventory [some experiences with the Beck depression inventory]. Gedrag, 13, 13-21.

Bouman, T. K., \& Meijer, K. J. (1999). A preliminary study of worry and metacognitions in hypochondriasis. Clinical Psychology and Psychotherapy, 6, 96-101.

Bouman, T. K., \& Polman, A. (2007). Group psychoeducation for hypochondriasis: A waitlist controlled study. Manuscript submitted for publication.

Buwalda, F. M., Bouman, T. K., \& Van Duijn M. A. J. (2007). Psychoeducation for hypochondriasis: A comparison of a cognitive-behavioural approach and a problem-solving approach. Behaviour Research and Therapy, 45(5), 887899.

Cartwright-Hatton, S., \& Wells, A. (1997). Beliefs about worry and intrusions: The meta-cognitions questionnaire and its correlates. Journal of Anxiety Disorders, 11(3), 279-296.

DiNardo, P. A., Brown, T. A., \& Barlow, D. H. (1994). Anxiety disorders interview schedule for DSM-IV: Life time version. Albany: Graywind.

Flavell, J. H. (1979). Metacognition and cognitive monitoring: A new area of cognitive-developmental inquiry. Americal Psychologist, 34(10), 906-911.

Kellner, R. (1986). Somatization and hypochondriasis. New York: Praeger.

Nelson, T. O., \& Narens, L. (1990). Metamemory: A theoretical framework and some new findings. In G. H. Bower (Ed.), The psychology of learning and motivation (Vol. 26, pp. 125-173). New York: Academic Press.

Papageorgiou, C., \& Wells, A. (2003). An empirical test of a clinical metacognitive model of rumination and depression. Cognitive Therapy and Research, 27(3), 261-273.

Pilowsky, I. (1967). Dimensions of hypochondriasis. British Journal of Psychiatry, 113, 89-93.

Raudenbush, S. W. (1997). Statistical analysis and optimal design for cluster randomized trials. Psychological Methods, 2(2), 173-185.

Roussis, P., \& Wells, A. (2006). Post-traumatic stress symptoms: Tests of relationships with thought control strategies and beliefs as predicted by the metacognitive model. Personality and Individual Differences, 40, 111-122.

Salkovskis, P. M. (1989). Somatic problems. In K. Hawton, P. M. Salkovskis, J. Kirk, \& D. M. Clark (Eds.), Cognitive behaviour therapy for psychiatric problems. A practical guide. Oxford: Oxford Medical Publications.

Salkovskis, P. M., \& Warwick, H. M. C. (1986). Morbid preoccupations, health anxiety and reassurance: A cognitivebehavioural approach to hypochondriasis. Behaviour Research and Therapy, 24(5), 597-602.

Snijders, T., \& Bosker, R. (2000). Multilevel analysis: An introduction to basic and advanced multilevel modeling. London: SAGE Publications.

Stern, R., \& Fernandez, M. (1991). Group cognitive and behavioural treatment for hypochondriasis. British Medical Journal, 303, 1229-1231.

Van der Ploeg, H. M., Defares, P. B., \& Spielberger, C. D. (1980). Handleiding bij de Zelfbeoordelingsvragenlijst $(Z B V)$ [manual for the STAI]. Lisse: Swets \& Zeitlinger.

Visser, S. (2000). Hypochondriasis: Assessment and treatment. Dissertation: The Netherlands: University of Groningen.

Warwick, H. M. C., \& Salkovskis, P. M. (1989). Cognitive and Behavioural characteristics of primary hypochondriasis. Scandinavian Journal of Behaviour Therapy, 18(2), 8592.

Warwick, H. M. C., \& Salkovskis, P. M. (1990). Hypochondriasis. Behaviour Research and Therapy, 28, 105.

Wells, A. (1999). A metacognitive model and therapy for generalized anxiety disorder. Clinical Psychology and Psychotherapy, 6, 86-95.

Wells, A. (2000). Emotional disorders and metacognition: Innovative cognitive therapy. New York: John Wiley \& Sons Ltd.

Wells, A., \& Carter, K. (2001). Further tests of a cognitive model of generalized anxiety disorder: Metacognitions and worry in GAD, panic disorder, social phobia, depression, and nonpatients. Behavior Therapy, 32, 85-102.

Wells, A., \& Matthews, G. (1994). Attention and emotion: A clinical perspective. Hove: Erlbaum.

Wells, A., \& Papageorgiou, C. (1998). Relationships between worry, obsessive-compulsive symptoms, and meta-cognitive beliefs. Behaviour Research and Therapy, 36, 899913.

Wells, A., \& Sembi, S. (2004). Metacognitive therapy for PTSD: A core treatment manual. Cognitive and Behavioral Practice, 11, 365-377. 